\title{
APPLICATION OF RAMAN SPECTROSCOPY FOR NONDESTRUCTIVE EVALUATION OF COMPOSITE MATERIALS
}

\author{
Glenn A. Washer ${ }^{1}$, Thomas M.B. Brooks ${ }^{1}$ and Regor Saulsberry ${ }^{2}$ \\ ${ }^{1}$ Department of Civil and Environmental Engineering \\ University of Missouri-Columbia, Columbia, MO 65211 \\ Tel: (573) 884-0320 \\ Email: washerg@missouri.edu \\ Email: tmb4bd@mizzou.edu \\ ${ }^{2}$ Project Manager \\ NASA - White Sands Test Facility \\ Las Cruces, NM 88012
}

\begin{abstract}
This paper will present an overview of efforts to investigate the application of Raman spectroscopy for the characterization of Kevlar materials. Raman spectroscopy is a laser technique that is sensitive to molecular interactions in materials such as Kevlar, graphite and carbon used in composite materials. The overall goal of this research reported here is to evaluate Raman spectroscopy as a potential nondestructive evaluation (NDE) tool for the detection of stress rupture in Kevlar composite over-wrapped pressure vessels (COPVs). Characterization of the Raman spectra of Kevlar yarn and strands will be presented and compared with analytical models provided in the literature. Results of testing to investigate the effects of creep and hightemperature aging on the Raman spectra will be presented.
\end{abstract}

Keywords: Raman spectroscopy, stress rupture, Kevlar, NDE

\section{INTRODUCTION}

There are concerns about the long-term behavior of polymer materials such as Kevlar that can be susceptible to aging effects, environmental degradation and stress rupture. Stress rupture is of particular concern in applications such as composite over-wrapped pressure vessels (COPVs), where the Kevlar over-wrap may be maintained at elevated stress levels for extended periods of time. Vessels of this design are utilized to hold various gases on NASA vehicles, and there are concerns that aging effects and stress rupture could effect the performance characteristics of these vessels. One approach to mitigating the risks associated with aging materials is to develop nondestructive evaluation (NDE) technologies that can detect the onset of materials degradation. The overall goal of the research reported here is to evaluate Raman spectroscopy as a potential NDE tool for the detection of stress rupture.

Raman spectroscopy is the measurement of the intensity and frequency of photons inelastically scattered from molecules, where the energy of the photon is shifted from the incident energy due to change from the vibrational energy of the molecule. The frequency shifts 
are dependent upon the specific molecular geometry of the material, and are independent of the incident photon frequency. A certain material could have many shifts in frequency from the incident photon, revealing a Raman spectrum. Figure 1 shows the spectrum of Kevlar yarn. As shown in the figure, there are a number of peaks (Raman bands) in the spectra, each relating to a vibrational mode of the Kevlar molecular structure.

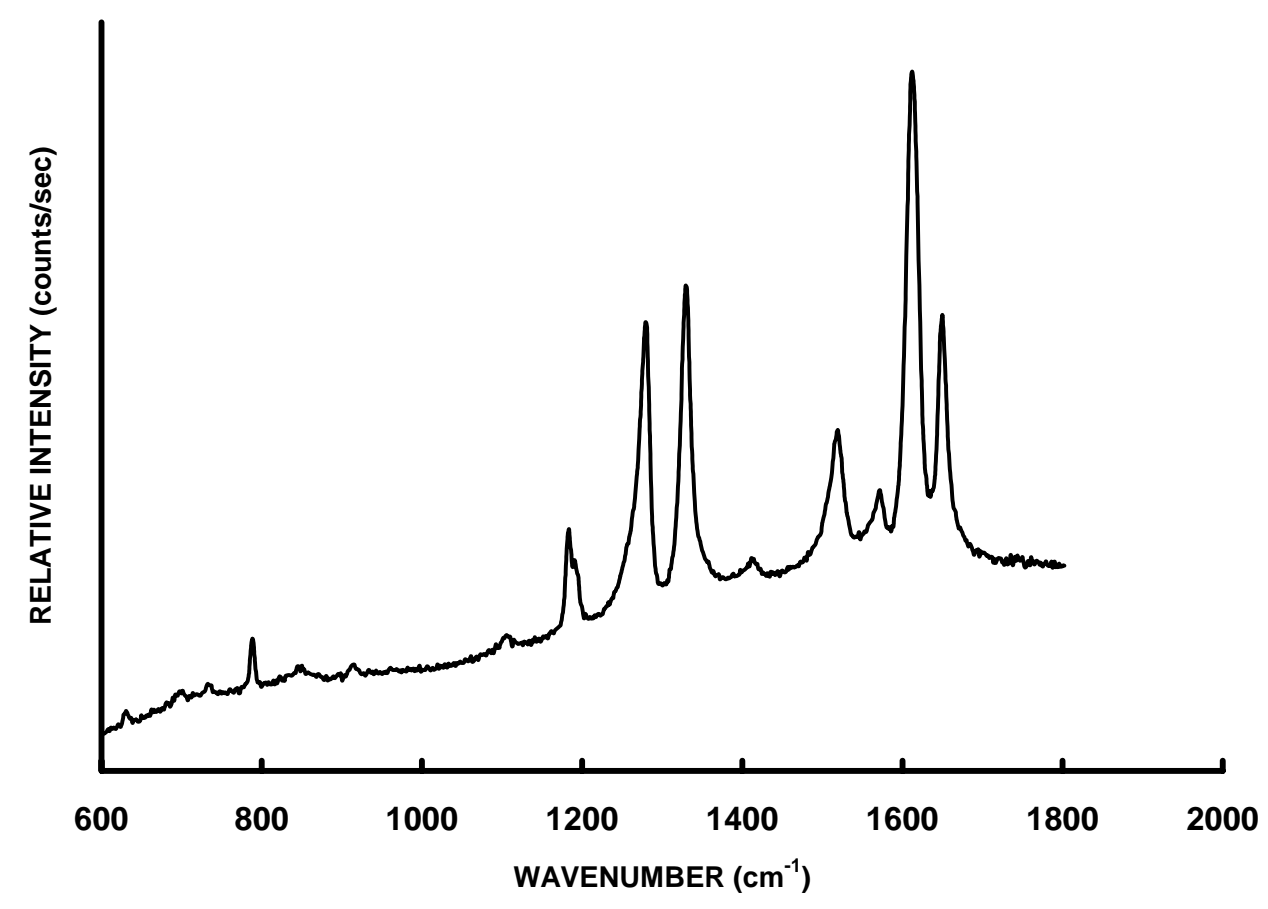

Figure 1: Raman spectrum of Kevlar 49.

Because Kevlar has a well defined Raman response, there have been several studies performed that characterize the structure of the polymer material through its Raman response and evaluate the material under applied strain[1-8]. Many previous studies have analyzed the behavior of a peak or band at $\sim 1613 \mathrm{~cm}^{-1}$; a band that has high sensitivity to applied strain. This band, as well as other bands in the spectrum, shifts when strain is applied to the polymer. Analyses of Kevlar by Penn[4] has determined a series of well defined peaks, or bands, ranging from 600 to $1700 \mathrm{~cm}^{-1}$. Each of these bands can be related to vibrational properties of the polymer.

\section{EXPERIMENTS}

Raman spectroscopy was conducted on a series of Kevlar 49 yarn, strand and composite material. This included a sample of virgin (unaged) Kevlar yarn, virgin strand (with resin), and strand that was creep-tested at elevated temperatures under an applied stress of $65 \%$ of the tensile strength. Composite sections removed from COPV specimens were also evaluated, including Kevlar composite materials aged under pressure for 17 years at $175^{\circ} \mathrm{F}$. Raman spectra 
were obtained and evaluated using a micro-Raman system with incident laser wavelengths ranging from $488 \mathrm{~nm}$ to $752 \mathrm{~nm}$, and a Fourier transform (FT) Raman system with a wavelength of $1064 \mathrm{~nm}$.

\section{RESULTS}

The primary results to date indicate that the Raman spectra detailed in the literature can be evaluated using a range of wavelength from $488 \mathrm{~nm}$ to $1064 \mathrm{~nm}$. Analyses were conducted of the obtained spectra compared with spectra described in the literature[4, 9]. Matching experimental peak values with those provided in the literature, it was found that in the range of 600 to $1700 \mathrm{~cm}^{-1}$ there was significant agreement. Table 1 lists the theoretical peaks compared to the experimental peaks. Using the FT Raman system with an incident laser wavelength of $1064 \mathrm{~nm}$, all theoretically predicted peaks could be identified. There was slightly less agreement using an incident wavelength of $647 \mathrm{~nm}$, finding 13 out 14 peaks predicted by Penn and Milanovich and 13 out 15 peaks predicted by Kim et al. There was significantly less agreement with an incident laser of $488 \mathrm{~nm}$. It is interesting to note a peak was found at $\sim 915 \mathrm{~cm}^{-1}$ using the 647 and $1064 \mathrm{~nm}$ lasers, but was absent in the literature.

\begin{tabular}{|c|c|c|c|}
\hline \multicolumn{2}{|c|}{ Theoretical } & \multicolumn{2}{c|}{ Kevlar strand } \\
\hline $\begin{array}{c}\text { Penn and } \\
\text { Milanovich } \\
\text { Peaks }\end{array}$ & $\begin{array}{c}\text { Kim et al. } \\
\text { Peaks }\end{array}$ & & \\
\hline $\mathbf{6 3 2}$ & $\mathbf{6 4 7} \mathbf{~ n m )}$ & (1064 nm) \\
$\mathbf{6 9 8}$ & $\mathbf{6 9 4}$ & 631 & 630 \\
$\mathbf{7 3 4}$ & $\mathbf{7 2 5}$ & 700 & 695 \\
$\mathbf{7 8 9}$ & $\mathbf{7 7 3}$ & 789 & 732 \\
- & $\mathbf{8 5 3}$ & - & 787 \\
- & - & 915 & 845 \\
$\mathbf{1 1 0 4}$ & $\mathbf{1 1 0 6}$ & 1105 & 1108 \\
$\mathbf{1 1 8 7}$ & $\mathbf{1 1 8 7}$ & 1184 & 1186 \\
$\mathbf{1 1 9 2}$ & $\mathbf{1 1 8 8}$ & - & 1194 \\
$\mathbf{1 2 7 9}$ & $\mathbf{1 2 8 3}$ & 1280 & 1283 \\
$\mathbf{1 3 3 1}$ & $\mathbf{1 3 3 2}$ & 1330 & 1332 \\
$\mathbf{1 4 0 9}$ & $\mathbf{1 4 0 0}$ & 1411 & 1417 \\
$\mathbf{1 5 1 8}$ & $\mathbf{1 5 1 6}$ & 1518 & 1521 \\
$\mathbf{1 5 7 0}$ & $\mathbf{1 5 6 7}$ & 1571 & 1574 \\
$\mathbf{1 6 1 5}$ & $\mathbf{1 6 1 5}$ & 1613 & 1615 \\
$\mathbf{1 6 4 9}$ & $\mathbf{1 6 5 4}$ & 1649 & 1651 \\
\hline
\end{tabular}

Table 1. List of theoretical peaks compared to the experimental peaks.

Characteristics of the Raman spectra that have been investigated include shifts in Raman peak values, changes in full width at half maximum (FWHM) ratios of Raman bands, and normalized intensity variations. Creep and fleet leader samples were evaluated to detect any changes in the Raman spectra compared to virgin samples. Laser wavelengths of 647 and 1064 
nm were used for all Raman spectra analyses since the spectra of these wavelengths provides the best depiction of the data.

No peak shifting has been detected relative to the $1613 \mathrm{~cm}^{-1}$ peak. Results of this analysis using a $647 \mathrm{~nm}$ laser are plotted in Figure 2 for virgin, creep, and fleet leader strands. The plots show that individual peaks have not shifted relative to each other. It has been found that there are slight variations in the locations of peaks when using different wavelengths of incident light, but this is believed to be a measurement artifact.

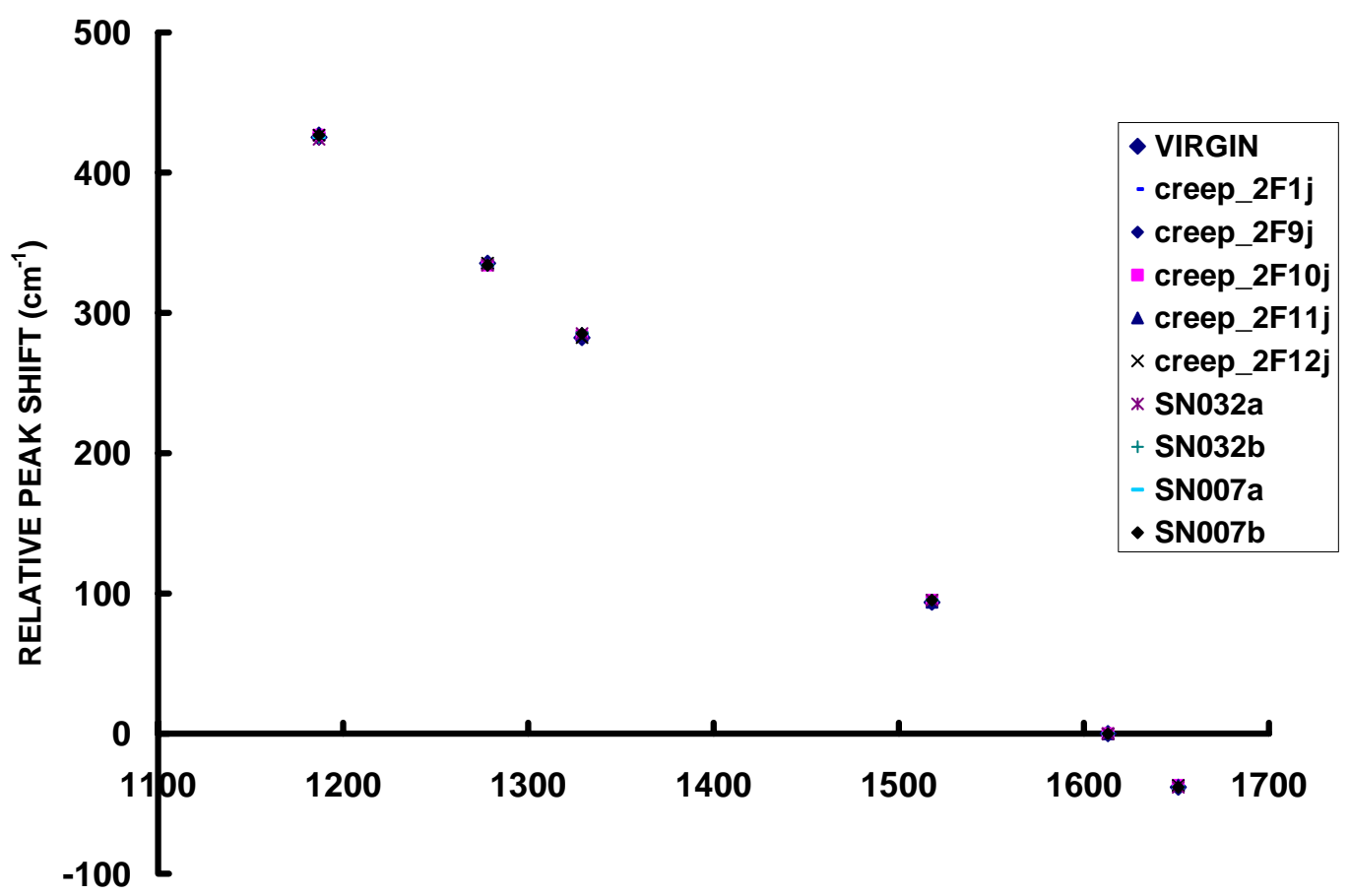

WAVENUMBER $\left(\mathrm{cm}^{-1}\right)$

Figure 2: Peak shifts of individual Raman bands using a 647 nm laser.

FWHM was analyzed for each major Raman band for the virgin, creep tested and the fleet leader samples. Each FWHM Raman band value was taken as a ratio to the FWHM of the 1278 $\mathrm{cm}^{-1}$ band for a particular spectrum. For the incident wavelengths of $647 \mathrm{~nm}$ and $1064 \mathrm{~nm}$, an increase in the FWHM ratios of the $1613 \mathrm{~cm}^{-1}$ band have been found for creep and fleet leader samples when compared to the virgin strands. Figure 3 shows the increase in FWHM ratio of the $1613 \mathrm{~cm}^{-1}$ band for the fleet leader samples to the virgin strands using the $647 \mathrm{~nm}$ incident laser.

The intensity ratios are also analyzed for each Raman band for all samples. Each intensity Raman band value was taken as a ratio to the intensity of the $1278 \mathrm{~cm}^{-1}$ band. Results to date have indicated that there is a variation in the peak intensity values for specimens that have been exposed to stress and elevated temperature, i.e. creep tested strands and fleet leader samples. It appears the intensity of the $1613 \mathrm{~cm}^{-1}$ band decreases for the creep and fleet leader samples 
compared to the virgin samples. This effect was found to be more pronounced in fleet leader samples that had been exposed to elevated temperatures.

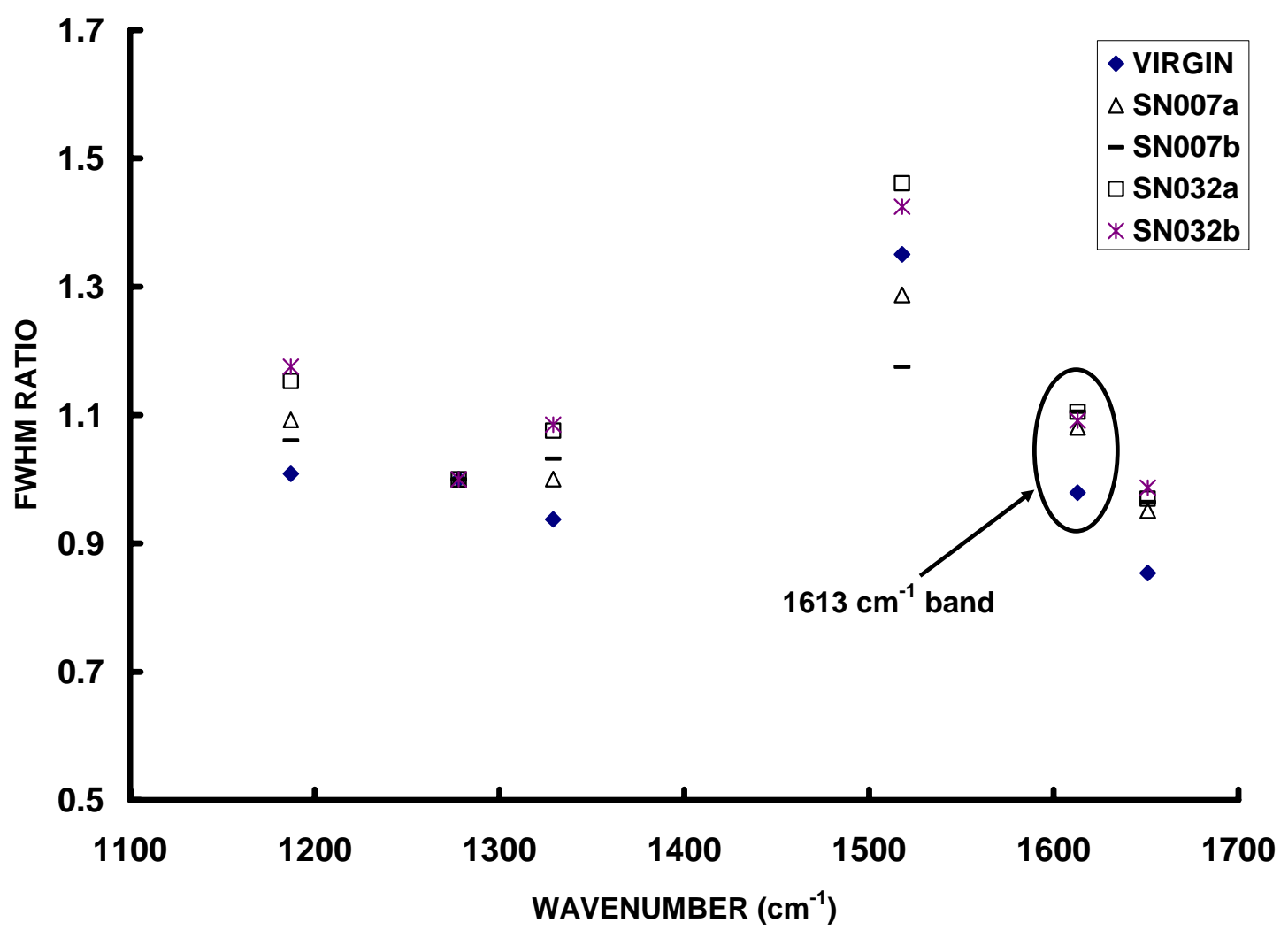

Figure 3: FWHM ratios of individual Raman bands using a 647 nm laser.

\section{CONCLUSIONS}

It has been observed in this study that aged strand materials from creep tested and fleet leader specimens have decreased normalized intensity ratios and increased FWHM ratios when compared with virgin strand specimens for the $1613 \mathrm{~cm}^{-1}$ band analyzed. In other words, the peaks are generally broader and of lower intensity than for virgin, undamaged specimens. Increases in FWHM may be attributed to defects in the fiber or defects being created in the fiber under stress[7]. On a molecular level, rod-like crystallites within the Kevlar distribute loading through hydrogen bonds between adjacent crystallites. Damage to crystallites due to aging phenomena may result in a redistribution of stresses on a molecular level, as damaged crystallites shed loading. Variant stresses within the material could result in photons being scattered over a slightly broader spectrum, producing peaks that are generally broader and lower in intensity. Additionally, previous research has suggested that hydrolysis in Kevlar may result in disruption to bonds between crystallites resulting in reduced intensity of the $\sim 1613 \mathrm{~cm}^{-1}$ band [8]. Exposure to high stresses and temperatures may be affecting the Kevlar materials examined in this study in a similar manner. 
It should also be noted that exposure to elevated temperatures and stresses did not have an effect on the proximate wavenumbers for the various peaks in the Raman spectrum of Kevlar. Very little variation in the wavenumbers for important bands such as the $1613 \mathrm{~cm}^{-1}$ indicates that these peak values, which vary as a function of strain, do not vary due to exposure to harsh environmental conditions of increased temperature and stress.

\section{REFERENCES}

1. Chang, C., Hsu, S. L., An Analysis of Strain-Induced Frequency Changes in Poly ( $p$ phenylene terephthalamide) Single Fibers. Macromolecules, 1990. 23: p. 1484-1486.

2. $\quad$ Andrews, M.C., Bannister, D.J., Young, R.J., The Interfacial Properties of Aramid/Epoxy Model Composites. Journal of Materials Science, 1996. 31: p. 3893-3913.

3. Galiotis, C., Robinson, I.M., Young, R.J., Smith, B., Batchelder, D. N., Strain Dependence of the Raman Frequencies of a Kevlar 49 Fiber. Polymer Communications, 1985. 26: p. 354-355.

4. $\quad$ Penn, L., Milanovich, F., Raman Spectroscopy of Kevlar 49 Fiber. Polymer, 1979. 20(1): p. 31-36.

5. Schadler, L.S., Galiotis, C., Fundamentals and Applications of Micro Raman Spectroscopy to Strain Measurements in Fiber Reinforced Composites. International Materials Reviews, 1995. 40(3): p. 116-133.

6. Kawagoe, M., Hashimoto, S., Nomiya, M., Morita, M., Qiu, J., Mizuno, W., Kitano, H., Effect of Water Absorption and Desorption on the Inerfacial Degradation in a Model Composite of an Aramid Fiber and Unsaturated Polyester Evaluated by Raman and FT Infra-red Microscopy. Journal of Raman Spectroscopy, 1999. 30: p. 913-918.

7. Prasad, K., Grubb, D.T., Deformation Behavior of Kevlar Fibers Studied by Raman Spectroscopy. Journal of Applied Polymer Science, 1990. 41: p. 2189-2198.

8. Stuart, B.H., A Fourier Transform Raman Study of Water Sorption by Kevlar-49. Polymer Bulletin, 1995. 35: p. 727-733.

9. $\quad$ Kim, P.K., Chang, C., Hsu, S.L., Normal Vibrational Analysis of a Rigid Rod Polymer: poly(p-phenylene terephthalamide). Polymer, 1986. 27(1): p. 34-46. 